\title{
A judicialização do acesso à Oxigenoterapia Domiciliar Prolongada no Sistema Único de Saúde.
}

\section{Judicialization of access to long-term home oxygen therapy in the Brazilian Publich Health System.}

\section{El acceso a la terapia de oxígeno judicialización Cuidado de la operación prolongada en el Sistema de Salud Púbica Brasileña.}

Laura Cordeiro Rodrigues ${ }^{1}$

Kimielle Cristina Silva ${ }^{2}$

Maria Leopoldina Castro Villa ${ }^{3}$

\begin{abstract}
RESUMO: O objetivo deste trabalho foi analisar as ações judiciais de oxigenoterapia domiciliar prolongada (ODP) recebidas pela Coordenação Geral de Atenção Domiciliar do Ministério da Saúde em 2015. Foi realizada pesquisa em revistas científicas brasileiras de Saúde Coletiva indexadas internacionalmente sobre os perfis clínico e epidemiológico dos indivíduos que mais necessitam da ODP e as políticas públicas e/ou dispositivos vigentes que a regulamentam em âmbito federal, associando o escopo desses documentos com o perfil da população que a utiliza. Foram analisados os municípios que possuem Serviço de Atenção Domiciliar (SAD) implantado e que possuem organização para o fornecimento da ODP. Quanto aos perfis clínico e epidemiológico foi verificado que a maioria dos demandantes era homens, idosos e com Doença Pulmonar Obstrutiva Crônica. Foram encontradas portarias que instituíram o Programa de Assistência Ventilatória Não Invasiva aos Portadores de Doenças Neuromusculares. Dos municípios com SAD, 69\% possuem fluxo para o fornecimento da ODP. As portarias existentes não são específicas para ODP e são restritivas aos indivíduos com doenças neuromusculares, fator que justifica as demandas judiciais recebidas, mostrando que são necessários dispositivos específicos no SUS para esta terapia e que seja mais abrangente que as já existentes.

Palavras-Chave: Oxigenoterapia, Judicialização da Saúde, Assistência Domiciliar, Direito à saúde, SUS.
\end{abstract}

1 Universidade Federal do Rio Grande do Sul, UFRGS, Brasil. E-mail: laura.crodrigues@hotmail.com. Rua dos Atleticanos, 215, apto 401, Milionários, Belo Horizonte - Minas Gerais - Cep:30620-060

2 Ministério da Saúde. E-mail: Kimielle@gmail.com

3 Secretaria de Saúde do Distrito Federal, Gerência de Atenção Domiciliar. E-mail: leocastro.df@terra.com.br 
ABSTRACT: The goal of this study was to analyze lawsuits filed against the General Office of Home Care of the Ministry of Health in 2015 to obtain home long-term oxygen therapy (LTOT). Internationally indexed Brazilian journals on collectivehealth were examined to determine the clinical and epidemiological profiles of individuals who need LTOT the most, as were current public policies and provisions regulating this therapy at a federal level. The scope of these policies was compared to the profile of the population using LTOT. Municipalities that hadimplemented Home Care Service (SAD) and the organized provision ofLTOT were also studied. In terms of the clinical and epidemiological profiles, it was determined that most plaintiffs were elderly men with Chronic Obstructive Pulmonary Disease. Regulations establishing the Non-invasive Assistance Program for Persons with Neuromuscular Diseases were also found. Out of the municipalities with $\mathrm{SAD}, 69 \%$ of themhave flowcharts for the provision of LTOT. Existing regulations are not specific to LTOT and are limited to individuals with neuromuscular diseases, which justifies the lawsuits filed, and shows that the Brazilian Healthcare System (SUS) requires specific provisions on this therapy that are more comprehensive than current ones.

Keywords: Oxygen therapy, The judicialization of health, Home Care, Right to health, SUS.

RESUMEN: El objetivo de este estudio fue analizar las demandas que solicitan oxigenoterapia domiciliaria prolongada (ODP) remitidas a la Coordinación General de Atención Domiciliaria del Ministerio de Salud en el año 2015. Se realizó una investigación con base en revistas científicas brasileñas de salud colectiva indexadas a nivel internacional sobre el perfil clínico y epidemiológico de las personas que más necesitan la ODP, y se llevó a cabo una búsqueda de las políticas públicas y disposiciones actuales que regulan la ODP a nivel federal. Se comparó el alcance de éstas con el perfil de la población que utilizaba la terapia. Por otra parte, se investigaron los municipios que cuentan con Servicio deAtención Domiciliar (SAD) yla provisión organizada de la ODP. En cuanto a los perfiles clínicos y epidemiológicos, se constató que la mayoría de los demandantes eran hombres de tercera edad con enfermedad pulmonaria crónica obstructiva. Fueron encontradas dos normas para establecer el Programa deAsistencia Ventilatoria No Invasiva para los Portadores de EnfermedadesNeuromusculares. De los municipios con SAD, el 69\% disponen de un diagrama de flujo para la provisión de la ODP. Las normas existentes no son específicas a la ODP y se limitan a las personas con enfermedades neuromusculares, factor que justifica las demandas recibidas, y pone de manifiesto que se requieren disposiciones en el SUS para esta terapia que sean más integrales que las existentes.

Palabras Clave: La terapia de oxígeno, La Judicialización de la salud, Cuidado del Hogar, Derecho a la Salud, SUS.

\section{INTRODUÇÃO}

O uso terapêutico do oxigênio iniciou por volta de 1922 e desde a década de 50 o cilindro de oxigênio já era prescrito no tratamento dos indivíduos com Doença Pulmonar Obstrutiva Crônica 
$(\mathrm{DPOC})^{1}$.

A oxigenoterapia consiste na administração do oxigênio em concentração e pressão maiores que as encontradas no meio ambiente para garantir o transporte adequado de oxigênio no sangue, reduzindo o trabalho da respiração ${ }^{2}$. Tem como objetivos a) reversão das alterações clínicas que cursam com insuficiência de oxigênio no sangue; b) manutenção da proteína que transporta o oxigênio (hemoglobina) e do volume de sangue bombeado pelo coração por minuto e; c) irrigação sanguínea adequada para a nutrição dos tecidos ${ }^{3}$.

Os primeiros estudos que subsidiaram as pesquisas atuais, bem como a consolidação no domicílio, foram do início dos anos 80. Os estudos demostraram que a Oxigenoterapia Domiciliar Prolongada (ODP) não só melhora a qualidade de vida como também aumenta a expectativa de vida dos indivíduos com doenças pulmonares que cursam com insuficiência de oxigênio no sangue, sobretudo a DPOC ${ }^{4,5}$.

Para a indicação da ODP é necessário o aparelho concentrador de oxigênio por, no mínimo, 15 horas diárias e o acompanhamento de profissionais de saúde ${ }^{6,7}$, o que faz com que seja considerada uma terapia de alto custo. Mesmo assim, autores apontam que é mais vantajosa quando comparada à internação hospitalar ${ }^{8}$, pois possibilita ao indivíduo permanecer em casa e próximo de seus familiares.

Estudo realizado sobre os custos da ODP em adultos demonstrou que o valor, em média, do aluguel do concentrador foi de $\mathrm{R} \$ 160,00$ ao mês. Já para cilindros, o cálculo dependeu da quantidade utilizada por indivíduo, sendo $\mathrm{R} \$ 110,00$ por cilindro9 . Em crianças o uso do concentrador em substituição ao cilindro possibilitou uma redução de 54\% nos custos dessa terapia ${ }^{8}$.

Entretanto, essas pesquisas não incluíram os gastos com a energia elétrica, que são custeados pelo individuo e/ou familiar. Verifica-se que há iniciativas do Estado que minimizam o valor do tratamento, como a Portaria MS/GM n ${ }^{\circ} 630$ de 2011 que instituiu a Tarifa Social de Energia Elétrica beneficiando indivíduos com renda mensal inferior a três salários mínimos. Porém, para serem beneficiados devem possuir doença ou deficiência (física, motora, auditiva, visual, intelectual e múltipla) e cujo tratamento, procedimento médico ou terapêutico necessite do uso contínuo de aparelhos, equipamentos ou instrumentos que, para o seu funcionamento, demandem consumo de energia elétrica ${ }^{10}$. A Portaria possibilita, portanto, a redução dos custos da terapia para as pessoas que dependem da ODP.

A Coordenação-Geral de Atenção Domiciliar da Secretaria de Atenção à Saúde do Ministério da Saúde (CGAD/SAS/MS) fomenta e coordena as ações de Atenção Domiciliar (AD) no âmbito do Sistema Único de Saúde (SUS) por meio do Programa Melhor em Casa.

A Portaria GM n ${ }^{\circ} 825$, de 25 de abril de $2016^{11}$, define a AD como: 
Modalidade de atenção à saúde integrada às Rede de Atenção à Saúde (RAS), caracterizada por um conjunto de ações de prevenção e tratamento de doenças, reabilitação, paliação e promoção à saúde, prestadas em domicílio, garantindo continuidade de cuidados ${ }^{11}$.

Trata-se de uma modalidade que propicia um cuidado mais humanizado, potente para produzir novas práticas de cuidados, pois o indivíduo estará em sua casa ou de familiares dentro de uma rotina conhecida e com mais privacidade ${ }^{12,13}$. E não é diferente no contexto da utilização da ODP e a AD tem um importante papel por possibilitar que as pessoas permaneçam em suas casas com mais qualidade de vida do que em um hospital.

Embora os manuais do Ministério da Saúde, como os Cadernos de Atenção Domiciliar ${ }^{10}$, abordem os critérios clínicos de indicação e admissão ao Programa de Oxigenoterapia Domiciliar, verifica-se que não há uma regulamentação para o fornecimento desse insumo. Tal fato contribui para a judicialização da ODP e para a assistência domiciliar no SUS.

A judicialização da saúde caracteriza-se por reivindicações e modos de atuação do Poder Judiciário para a garantia dos direitos de cidadania amplamente afirmados nas leis internacionais e nacionais. Esse fenômeno envolve aspectos políticos, sociais, éticos e sanitários que vão muito além de seu componente jurídico e de gestão de serviços públicos de ações judiciais em que há solicitação de insumos, procedimentos e medicamentos ${ }^{14}$.

É um fenômeno recente e de grande relevância que vem ocorrendo no Brasil e em outros países, além de envolver inúmeros interessados, como o cidadão, o mercado tecnológico da saúde, as associações e a indústria farmacêutica ${ }^{15}$. No Brasil surgiu na década de 90 a partir dos pedidos de medicamentos antirretrovirais para a Síndrome da Imunodeficiência Adquirida (SIDA/AIDS) e representou importante papel como via alternativa do cidadão ao acesso a medicamentos no SUS, ou seja, ao direito integral à saúde ${ }^{16}$.

Várias são as razões que explicam a judicialização, mas para Barroso ${ }^{17}$ são três principais. A primeira refere-se à redemocratização do Estado que propiciou aos indivíduos o exercício da sua cidadania e a busca do Judiciário para protegerem seus interesses, além da expansão das Defensorias Públicas para diversas partes do país; a segunda destaca a saúde como um direito na Constituição Federal, o que a transforma potencialmente em pretensão jurídica ${ }^{17} \mathrm{e}$; a terceira é o sistema brasileiro de constitucionalidade que permite o questionamento de qualquer lei perante o juiz ou tribunal ${ }^{17}$.

Esse fenômeno não foi espontâneo, tampouco isolado, mas estimulado inicialmente por movimentos sociais de saúde organizados e fortalecidos pelos Conselhos de Saúde. Nos Conselhos participam entidades de proteção dos direitos de pessoas com algum agravo ou doença, como diabetes, hemofilia, dentre outros, tendo papel historicamente importante na judicialização da 
saúde no Brasil ${ }^{18}$.

Sabe-se da relevância que o assunto tem adquirido nos últimos anos, principalmente do impacto no orçamento público, sendo a judicialização um desafio para a gestão de saúde e para o sistema de justiça. É considerada um evento multifacetado, questão ampla e diversa de reclame de bens e direitos como insumos, instalações, medicamentos, assistência à saúde, alimentos, entre outras demandas a serem protegidas pelo princípio do direito à saúde ${ }^{19}$. Além de expor os limites e possibilidades institucionais, instiga a produção de respostas efetivas pelos agentes públicos do sistema de saúde e do sistema de justiça ${ }^{20}$.

A Constituição Federal de 1988 introduziu também o chamado federalismo solidário que impôs o cuidado da saúde como competência comum à União, aos Estados, ao Distrito Federal e aos Municípios, embora tenha optado pela municipalização na execução dos serviços de saúde ${ }^{21,18,9}$.

O fato da judicialização ser utilizada como ferramenta de afirmação da saúde como direito, alguns autores a evidenciam como o reconhecimento de pretensões individuais a prestações positivas do Estado e que as lacunas das políticas públicas e as falhas na sua execução ${ }^{22,23}$ acarretam impacto importante no sistema de saúde, tanto para a gestão quanto para o sistema de financiamento do SUS.

Há, no entanto, estudos que mostram efeitos negativos que podem ser divididos em dois aspectos principais. $\mathrm{O}$ primeiro aponta que o deferimento de pedidos judiciais pode aprofundar as iniquidades de acesso à saúde, infringindo um dos princípios do SUS. Isso ocorre porque as ações individuais não são estendidas aos demais portadores da mesma condição patológica que poderiam se beneficiar com o objeto da ação judicial, bem como o favorecimento daqueles que têm maior possibilidade de acesso ao Poder Judiciário para veicular sua demanda em detrimento dos que não o possuem ${ }^{24}$. $\mathrm{O}$ segundo aspecto refere-se à segurança do indivíduo em razão das possíveis prescrições inadequadas, em especial na prescrição de novos medicamentos e/ou novas indicações terapêuticas para os quais as evidências científicas ainda não se encontram bem estabelecidas ${ }^{25}$.

Apesar de todos os problemas que a judicialização acarreta à gestão do SUS, vários autores a consideram como uma forma legítima de garantir o exercício do direito à saúde com o argumento que este direito é inviolável, independentemente de questões políticas e orçamentárias ${ }^{16,1}$. Além da possibilidade de ser vista como um diagnóstico, dos insumos e/ou tratamentos, que a população não consegue acessar conforme os princípios do SUS e que, a partir disso, pode produzir estudos que subsidiem ações que visem a redução dos processos e a garantia da saúde como direito social. Dentre essas ações, destaca-se a criação de políticas públicas que orientem a implantação e o fornecimento de serviços de saúde.

Diante desse cenário e da pouca produção científica existente acerca da judicialização da ODP, o presente estudo buscou compreender as demandas judiciais relativas à oxigenoterapia domiciliar 
prolongada recebidas pelo Ministério da Saúde e encaminhadas à CGAD/SAS/MS para emissão de parecer técnico no período de 2012 a 2015.

\section{METODOLOGIA}

Trata-se de um estudo exploratório e descritivo que buscou conhecer sobre a judicialização da oxigenoterapia domiciliar prolongada e a sua conexão com a carência de normas que a regulamentam, tema esse ainda pouco estudado.

A pesquisa teve duração de abril a outubro de 2015. Durante esse período foram analisados os dados referentes aos processos judiciais recebidos pela Coordenação nos anos de 2012 a abril de 2015.

O primeiro passo foi realizar uma pesquisa nas bases de dados de relevância para a produção do conhecimento em saúde sobre o perfil das pessoas que utilizam a ODP: PubMed, Bireme e SciELO. Para isso, utilizou-se o descritor Oxigenoterapia Domiciliar Prolongada.

O segundo passo foi a busca das políticas públicas e/ou dispositivos vigentes em âmbito federal que regulamentam a ODP no sítio eletrônico Saúde Legis do Ministério da Saúde, associando o escopo dessas políticas e/ou dispositivos ao perfil da população que utiliza a ODP.

Já o terceiro passo foi a análise do banco de dados da CGAD/SAS/MS referentes ao fornecimento da ODP, onde buscou-se os municípios com organização do cuidado, fluxograma, protocolos ou linhas de cuidado para os indivíduos que utilizam a ODP. Esses dados foram obtidos a partir de um questionário enviado por e-mail pela CGAD/SAS/MS a todos os gestores de AD dos municípios brasileiros, a fim de conhecer a forma de funcionamento dos Serviços de Atenção Domiciliar (SAD), contendo as seguintes perguntas: o município possui organização para fornecimento da ODP? Se sim, a regulação é feita pelo SAD?

Quanto à ética em pesquisa, este artigo baseou-se em um trabalho de conclusão de curso e teve como subsídio a resolução do Conselho Nacional de Saúde no 466 de 12 de dezembro de 2012, cumprindo os requisitos de respeito à condição humana, autonomia, não-maleficência, justiça e equidade, dentre as outras exigências explícitas na Resolução. Não foi necessária submissão ao Comitê de Ética em Pesquisa, pois foram utilizados dados secundários públicos ${ }^{26}$, subsidiada pela Lei de Acesso à Informação no 12.527 de 18 de novembro de $2011^{27}$. 


\section{RESULTADOS}

As solicitações de ODP representaram 30,4\% dos processos judiciais, ou seja, 24 dentre os 79 processos judiciais no período de 2012 a 2015.

\section{Perfis clínico e epidemiológico}

Quanto aos perfis clínico e epidemiológico, os indivíduos que dependem desta tecnologia são, conforme publicação de Floriano et al (2012) ${ }^{32}$, adultos e quanto à idade, Lacerda $(2013)^{6}$ apontou que a maioria era idoso. E os estudos de Watanabe et al $(2015)^{9}$, por sua vez, evidenciaram que a maioria era do sexo masculino, tabagistas e ex-tabagistas ${ }^{32,6,9}$.

De acordo com estudos da American Thoracic Society $(1995)^{2}$ e da Sociedade Brasileira de Pneumologia e Tisiologia (2000 e 2004) ${ }^{3}$, a principal doença pulmonar, em adultos, que cursa com insuficiência crônica de oxigênio no sangue e necessita da ODP é a Doença Pulmonar Obstrutiva Crônica (DPOC) $)^{2,3,33}$, corroborando com dado da DPOC ser o diagnóstico mais citado nos processos recebidos pela $\mathrm{CGAD} / \mathrm{SAS} / \mathrm{MS}$.

Já em crianças, Lynn (2011) apontou outros agravos mais prevalentes no uso da ODP, como a fibrose cística ${ }^{34}$.

Portanto, hoje a ODP é considerada o principal tratamento não farmacológico para os indivíduos portadores da DPOC e é indicada para os portadores da doença em estágio avançado 3 .

\section{Normativas}

Foram encontradas duas normativas vigentes do Ministério da Saúde: a Portaria MS/GM n ${ }^{\circ}$ 1370, de 3 de julho de $2008^{28}$, que instituiu o Programa de Assistência Ventilatória Não Invasiva aos Portadores de Doenças Neuromusculares, e a Portaria MS/SAS no 370 , de 4 de julho de $2008{ }^{29}$, que orienta a organização e a implantação do Programa, define o rol de doenças a serem contempladas e estabelece critérios técnicos de implantação do Programa e da Assistência Ventilatória aos portadores de doenças neuromusculares que dela necessitam.

Verificou-se que após a consulta pública, em 4 de julho de 2012, o procedimento foi incorporado ao SUS para o tratamento da DPOC, por meio da $1^{\text {a }}$ Reunião Extraordinária do Plenário da Comissão Nacional de Incorporação de Tecnologias no SUS (CONITEC) ${ }^{30}$.

No ano de 2013, o Ministério da Saúde aprovou a Portaria no 609, que normatiza o Protocolo Clínico e Diretrizes Terapêuticas (PCDT) para a DPOC, que aborda o conceito geral da doença, os critérios de diagnóstico, de inclusão e de exclusão, as diretrizes para o tratamento e os mecanismos de regulação, controle e avaliação ${ }^{31}$. O PCDT é de caráter nacional e deve ser utilizado pelas secretarias de saúde dos Estados, do Distrito Federal e dos Municípios na regulação do acesso 
assistencial, autorização, registro e ressarcimento dos procedimentos correspondentes. Dentre os procedimentos propostos para o tratamento da DPOC encontra-se a Oxigenoterapia Domiciliar Prolongada, com os critérios para a sua prescrição ou não.

\section{Fornecimento de ODP pelos municípios, estados e Distrito Federal}

No que se refere à organização da atenção à saúde dos indivíduos que necessitam da ODP no âmbito municipal ou estadual, dos 318 SAD implantados, ou seja, em funcionamento, cerca de $38,7 \%(\mathrm{~N}=123)$ forneceram dados para a Coordenação. Desses, 69,10\% $(\mathrm{N}=85)$ informaram que possuem uma organização para o fornecimento da ODP. Verificou-se que na maioria dos municípios $(66 \%)$ a regulação não é realizada pelo SAD.

GRÁFICO 1. Percentual de Municípios que possuem serviço organizado para o fornecimento de ODP em 2015, por região.

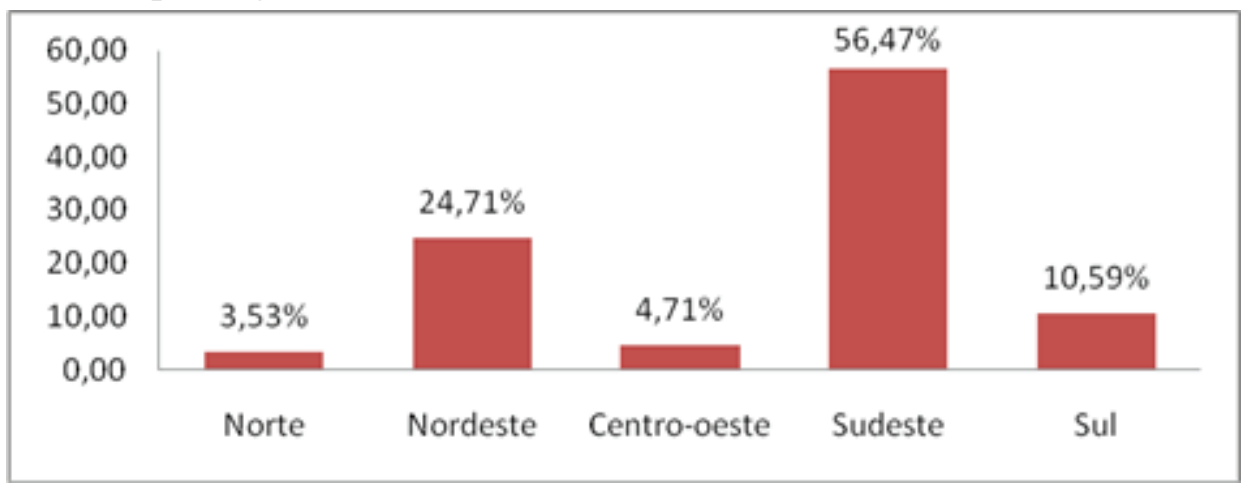

Verificou-se que cinco estados possuem programa estadual para o fornecimento da ODP, sendo que um destaca-se por ter como foco apenas os indivíduos egressos de hospitais estaduais (GRÁFICO 2).

GRÁFICO 2 Percentual de Estados que possuem programas estaduais de fornecimento de ODP em 2015, por região.

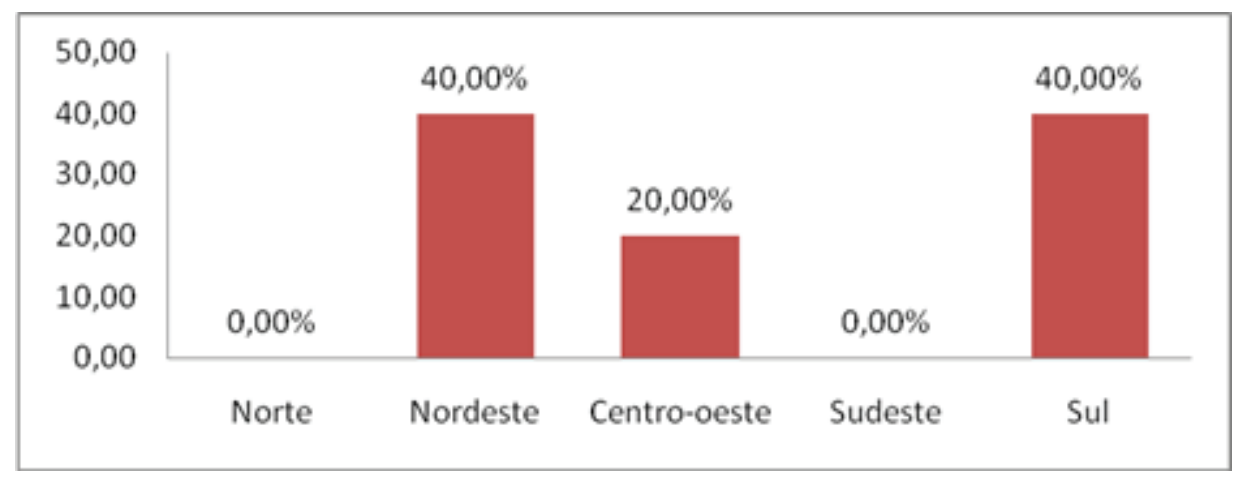

\section{DISCUSSÃO}

Os resultados encontrados mostram que não há portarias do Ministério da Saúde que regulamentam a ODP para indivíduos em geral.

A existência de vários documentos, como o relatório da CONITEC, que incorporou a ODP ao 
SUS, bem como o PCDT e o Caderno de Atenção Domiciliar volume II, que trazem a indicação do seu uso, indicam que o Ministério da Saúde tem buscado atender as demandas da população, reconhecendo a eficácia desta terapêutica ${ }^{30,31,10}$.

É importante ressaltar que o Caderno de Atenção Domiciliar vol. II é abrangente quanto à indicação da terapia, não restringindo a um grupo específico de patologias. Já PCDT de DPOC abarca o perfil clínico das pessoas que mais necessitam da ODP, diferentemente das Portarias $\mathrm{n}^{\circ}$ 1370 e n 370 , ambas de $2008^{10,31,28,29}$.

A existência destes documentos aponta que esta terapia já deveria estar disponível no SUS e, conforme o preceito da municipalização, os municípios deveriam ofertá-la, o que demonstra uma vulnerabilidade do sistema de saúde.

Esta fragilidade na organização da oferta da ODP pelo SUS nos municípios juntamente com a falta de diretrizes da União quanto ao fornecimento da terapia preocupam, pois verifica-se o aumento do número de indivíduos que necessitam da ODP em diversas localidades. Um estudo multicêntrico realizado em cinco grandes cidades da América Latina encontrou a prevalência de $15,8 \%$ de DPOC em indivíduos com 40 anos ou mais ${ }^{35}$. Já a prevalência mundial de DPOC, apontada pela Global Initiative for Chronic Obstructive Lung Disease (GOLD), para todas as idades foi de $11,6 / 1000$ para homens e 8,8/1000 para mulheres ${ }^{36}$.

A demanda por ODP também tem prevalência importante, sobretudo entre os indivíduos acompanhados em domicílio. No Distrito Federal, por exemplo, um estudo realizado entre os anos de 2012 e 2013, que analisou o prontuário de 857 indivíduos, encontrou prevalência de $31,5 \%$ de utilização da $\mathrm{ODP}^{37}$.

Observa-se que o uso da ODP foi mais frequente em idosos, conforme os estudos de Moraes et al (2010) ${ }^{38}$. É importante ressaltar que com a progressão da idade as alterações fisiológicas não culminam em adoecimento, porém, tornam os indivíduos mais susceptíveis a processos de perda de capacidade funcional, o que exige cuidados mais atentos ao processo de envelhecimento e às patologias mais prevalentes ${ }^{38}$.

Considerando que a ODP é uma terapia consolidada cientificamente e com uma boa relação de custo-benefício tanto do ponto de vista financeiro quanto social, pois permite aos indivíduos viverem nas suas casas e próximos de seus familiares, poderia haver maior esforço da esfera pública para dar acesso a todos que dela necessitam.

De acordo com os resultados deste estudo, as portarias sobre o fornecimento da ODP vigentes não alcançam o perfil clínico dos indivíduos que mais necessitam, o que indica que há necessidade de os serviços se estruturarem para fornecer ou ampliar o leque de oferta da terapia. Um exemplo

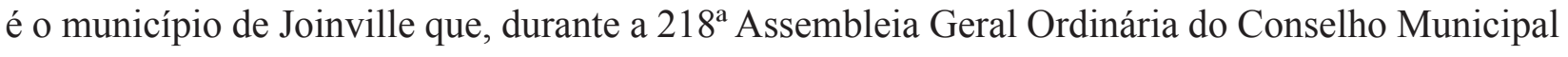
de Saúde em 2012, aprovou o credenciamento da Oxigenoterapia Prolongada Domiciliar, 
especificamente para pessoas com doença neuromuscular ${ }^{39}$. O que exemplifica o poder de indução de portarias e políticas federais para a organização e o fornecimento de serviços pelos municípios que, neste caso, são restritivas frente aos perfis clínico e epidemiológico encontrados neste estudo.

Mesmo que existam documentos que, em tese, garantam o acesso da população à terapia, os processos judiciais recebidos pela CGAD/SAS/MS evidenciam as fragilidades e falhas do sistema de saúde, no caso deste estudo, no fornecimento da ODP, mostrando que tais documentos podem ser insuficientes para induzir a organização dos municípios e estados na implementação de serviços estruturados para garantir o acesso à terapia aos cidadãos.

Com isso, parte da população fica sem acesso ao tratamento, mesmo a saúde estando assegurada como direito social na Constituição Federal. Além disso, a legislação do SUS possui princípios que também asseguram direito à saúde de forma integral, universal e equânime ${ }^{40}$, o que impõe que tal serviço faça frente às necessidades do usuário ${ }^{41}$.

Alguns autores têm demonstrado que grande parte dos municípios não possuem capacidade técnica e financeira para cumprirem sozinhos o preceito universalizador e o atendimento integral à saúde, o que os torna reféns da cooperação técnica e financeira dos Estados e da União ${ }^{18}$.

Quando esta cooperação técnica e financeira não acontece de forma adequada entre os entes federados, alguns municípios não conseguem garantir acesso a insumos e tratamentos. E o fato dos cidadãos terem mais conhecimento sobre seus direitos, mais acesso ao Poder Judiciário e pouco conhecimento acerca das políticas públicas de saúde e funcionamento do SUS contribui para o aumento das demandas judiciais.

Verifica-se que a maioria dos estados e municípios possui serviço de saúde organizado para fornecer a ODP, seja pela existência de fluxo, protocolo ou até mesmo portarias. Porém, em mais da metade dos municípios $(66,0 \%)$, a regulação do fornecimento não é feita pelo SAD.

Uma possível justificativa para tal resultado é de que existem alguns pré-requisitos para um município pleitear a implantação de um SAD, como a pactuação na Comissão Intergestores Bipartite (CIB), a retaguarda de hospital, a cobertura de Serviço de Atendimento Móvel de Urgência (SAMU) ou serviço semelhante e os critérios populacionais ${ }^{11}$, o que pode indicar que são municípios com serviços de saúde mais estruturados.

Além disso, apesar da portaria que regulamenta a $\mathrm{AD}$ permitir que municípios menores se agrupem para implantar o SAD, no período de elaboração deste estudo existiam apenas dois agrupamentos, ou seja, a maioria dos municípios com SAD tem mais de 20 mil habitantes, o que também pode justificar os dados de municípios que têm SAD e fornecem a ODP.

$\mathrm{O}$ caderno AD vol. $\mathrm{II}^{10}$ pode ter influenciado alguns serviços $\mathrm{AD}$ a organizarem o fornecimento da ODP com os recursos financeiros destinados a essas equipes ou até mesmo assumirem a 
regulação de programas, protocolos e fluxos já existentes.

Uma limitação do estudo foi que os dados referentes ao fornecimento da ODP pelos Estados, Distrito Federal e Municípios se restringiram aos que possuem SAD implantado e que forneceram dados para a CGAD/SAS/MS. Além disso, os dados não são claros para distinguir se o fornecimento acontece para indivíduos em geral ou subsidiado pelas Portarias $n^{\circ} 1370$ e $n^{\circ} 370$ de 2012, o que limita o fornecimento para pessoas com doenças neuromusculares.

Pode-se pressupor que municípios com o Programa Melhor em Casa possuem serviços de saúde mais estruturados, já que a adesão ao Programa depende de uma estrutura de saúde, porém, são necessários mais estudos sobre a epidemiologia da utilização da ODP e que analisem os processos judiciais de forma estratificada para saber se os locais com maior número de demandas possuem ou não organização para o fornecimento da terapia, bem como estudos que analisem todos os municípios com e sem SAD, para então concluir se a solução seria a elaboração de uma política específica ou a criação de um dispositivo dentro de uma política já existente. Vale compreender melhor as organizações existentes nas esferas federal e estadual para que sirvam de modelo para subsidiar a formulação desta nova política ou dispositivo.

Sabendo do gasto elevado com as demandas judiciais pelos três entes federados e dos problemas causados na gestão dos serviços de saúde, o assunto tem sido muito debatido e tem ganhado destaque. No entanto, essa atenção ainda é discreta e com ênfase maior para os gastos do que para o fenômeno em si e o que ele pode indicar. Há, portanto, a necessidade de aproximação entre o sistema de saúde e o judiciário visando manter a integração permanente para discutir, refletir e pensar soluções conjuntas para este problema.

É necessário melhorar o diálogo entre os gestores de saúde, das três esferas de governo, e o Poder Judiciário, a fim de que sejam pensadas soluções conjuntas.

A fim de seguir os preceitos do federalismo solidário, faz sentido que a discussão seja partilhada entre a União, os Estados, o Distrito Federal e os Municípios. É, portanto, necessário discutir o tema em espaços compartilhados, como a Comissão Intergestores Tripartite (CIT), objetivando encontrar caminhos para a organização dos entes federados para o fornecimento da ODP, inclusive, garantindo o financiamento tripartite e evitando, assim, que os municípios assumam sozinhos o fornecimento da terapia.

\section{CONSIDERAÇÕES FINAIS}

Os resultados deste estudo evidenciam que as portarias vigentes não contemplam o perfil dos indivíduos que mais necessitam da ODP, o que pode justificar o elevado número de demandas judiciais recebidas pelo Ministério da Saúde solicitando esta terapia.

Sabendo-se que a judicialização pode acarretar problemas para a gestão e para o funcionamento 
dos serviços de saúde, mas também considerando que é uma forma dos cidadãos buscarem assegurar os direitos constitucionalmente conferidos, deve-se entender melhor o fenômeno e buscar maneiras de conter o seu crescimento. Faz-se necessário investir conjuntamente na garantia de acesso aos serviços de saúde e na melhoria e ampliação das políticas públicas de saúde, bem como na qualificação dos gestores, a fim de garantir sua execução.

Há, portanto, a necessidade de discutir de forma tripartite a judicialização da ODP com a finalidade de definir ações que subsidiem os municípios para o fornecimento de uma terapia que, teoricamente, já deveria ser ofertada aos usuários. É preciso definir os atores responsáveis e os fluxos para o fornecimento da ODP, uma vez que as políticas públicas se encontram dispersas em diversos atos normativos, sem uma sistematização clara e, muitas vezes, com trâmites que contrastam com as necessidades postas nos processos.

\section{AGRADECIMENTOS}

Agradecemos à Universidade Federal do Rio Grande do Sul (UFRGS) pela oportunidade de realizar a imersão no Ministério da Saúde por meio do Programa de Desenvolvimento Tecnológico e Extensão Inovadora em Atenção Básica e Educação em Saúde Coletiva, o que inspirou a realização deste trabalho.

\section{REFERÊNCIAS BIBLIOGRÁFICAS}

1. Machado, M et al. Judicialização do acesso a medicamentos no Estado de Minas Gerais, Brasil. Revista de Saúde Pública. 2011;45(3):590-8.

2. American Thoracic Society: ATS. Standards for the diagnosis and care of patients with chronic obstructive pulmonary disease. American Journal of Respiratory and Critical Care Medicine. 1995;152 (5): 77-120.

3. Sociedade Brasileira de Pneumologia e Tisiologia: SBPT. Oxigenoterapia domiciliar prolongada (ODP). Jornal Brasileiro de Pneumologia. 2000; 26(6).

4. Nocturnal Oxygen Therapy trial group: NOTT. Continuous or nocturnal oxygen therapy in hypoxemic chronic obstructive lung disease: a clinical trial. Annals of Internal Medicine. 1980; 93(3): 391-398.

5. Medical Research Council Working Party. Long term domiciliary oxygen therapy in chronic hypoxic corpulmonale complicating chronic bronchitis and emphysema. Lancet. 1981; 28(1): 681-686.

6. Lacerda, Z. Perfil dos indivíduos usuários de oxigenoterapia domiciliar prolongada do município de Goiânia. Pontifícia Universidade Católica de Goiás - Centro de estudos avançados e formação integrada- Especialização em fisioterapia cardiopulmonar, Goiânia, 2013. 
7. O'Rilley, P; Bailey, W. Long-term continuous oxygen treatment in chronic obstructive pulmonary disease: proper use, benefits and unresolved issues. CurrentOpinioninPulmonaryMedicine. 2007; 13(2):120-4.

8. Munhoz, A et al. Oxigenoterapia domiciliar prolongada em crianças e adolescentes: uma análise do uso clínico e de custos de um programa assistencial. Jornal de Pediatria. 2011; 87(1):13-18.

9. Watanabe, C. et al. Oxigenoterapia domiciliar prolongada: perfil dos usuários e custos. Revista de Enfermagem da UERJ. 2015; 23(1):95-101.

10. Brasil. Ministério da Saúde. Secretaria de Atenção à Saúde. Departamento de Atenção Básica. Caderno de atenção domiciliar, Volume 2, Brasília (DF): Ministério da Saúde, 2013 b.

11. Brasil. Ministério da Saúde. Portaria $n^{0}$ 963, de 27 de maio de 2013. Redefine a Atenção Domiciliar no âmbito do Sistema Único de Saúde (SUS). Diário Oficial da União 2013 a; 29 ago.

12. Silva, KL et al. Atenção domiciliar como mudança do modelo tecnoassistencial. Revista de Saúde Pública. 2010; 44(1):166-76.

13. Silva, KL et al. Challenges of homecare from the perspective of cost reduction/ expenditure optimization. Journal of Nursing UFLPE Online. 2014; 8(6): 1561-7.

14. Ventura, MV ET al. Judicialização da saúde, acesso à justiça e a efetividade do direito à saúde. Physis: Revista de Saúde Coletiva. 2010; 20(1):77-100.

15. Chieffi, AL; Barata, RCB. Ações judiciais: estratégia da indústria farmacêuticapara introdução de novos medicamentos. Revista de Saúde Pública. 2010; 44(3): 421-9.

16. Pepe, V et al. A judicialização da saúde e os novos desafios da gestão da assistência farmacêutica. Ciência e Saúde Coletiva. 2010; 15(5):2405-14.

17. Barroso, L. Judicialização, ativismo judicial e legitimidade democrática. Revista Eletrônica de Direito do Estado. 2012;5(1):23-32.

18. Santos, L; Terrazas, F. Judicialização da saúde no Brasil. Campinas: Saberes. 2014, 484 p.

19. Baptista, T; Machado, C; Lima, L. Responsabilidade do Estado e direito à saúde no Brasil: um balanço da atuação dos Poderes. Ciência e Saúde Coletiva. 2009; 14: 3, 829-39.

20. Brasil. Ministério da Saúde. Secretaria de Vigilância em Saúde. Programa Nacional de DST e AIDS. O Remédio via Justiça: um estudo sobre o acesso a novos medicamentos e exames em HIV/aids no Brasil por meio de ações judiciais / Ministério da Saúde, Secretaria de Vigilância em Saúde, Programa Nacional de DST e Aids. Brasília (DF): Ministério da Saúde, 2005. 
21. Brasil. Constituição (1988). Constituição da República Federativa do Brasil. 35. ed. Brasília (DF): Senado Federal, 2012 b.

22. Oliveira, M; Delduque, MC; Sousa, M; Mendonça, V. Judicialização da saúde: para onde caminham as produções científicas? Saúde e Debate. 2015; 38(10): 525-35.

23. Neto, A. Judicialização da saúde. Caderno Mídia e Saúde Pública. 2007; 2(1): 49-52.

24. Vieira, F; Zucchi, P. Distorções causadas pelas ações judiciais à política de medicamentos no Brasil. Revista de Saúde Pública. 2007; 41(2):214-22.

25. Chieffi, AL; Barata, R. Judicialização da política pública de assistência farmacêutica e equidade. Cadernos de Saúde Pública. 2009; 25(8):1839-49.

26. Brasil. Ministério da Saúde. Conselho Nacional de Saúde. Resolução no 466, de 12 de dezembro de 2012. Aprova normas regulamentadoras de pesquisas envolvendo seres humanos. Diário Oficial da União 2012 a; 13 jun.

27. Brasil. Lei $\mathrm{n}^{\mathrm{o}} 12.527$, de 18 de novembro de 2011. Regula o acesso a informações previsto no inciso XXXIII do art. 5o, no inciso II do § 3o do art. 37 e no § 20 do art. 216 da Constituição Federal; altera a Lei no 8.112, de 11 de dezembro de 1990; revoga a Lei no 11.111, de 5 de maio de 2005, e dispositivos da Lei no 8.159, de 8 de janeiro de 1991; e dá outras providências. Diário Oficial da União 2011; 18 nov.

28. Brasil. Ministério da Saúde. Portaria no 1370, de 03 de julho de 2008. Redefine a Atenção Domiciliar no âmbito do Sistema Único de Saúde (SUS). Institui o Programa de Assistência Ventilatória Não Invasiva aos Portadores de Doenças Neuromusculares. Diário Oficial da União 2008 a; 04 jul.

29. Brasil. Ministério da Saúde. Portaria no 370, de 04 de julho de 2008. Estabelece, na forma do anexo I desta portaria, o rol de doenças neuromusculares incluídas no programa de assistência ventilatória não invasiva aos portadores de doenças neuromusculares. Diário Oficial da União 2008 b; 07 jul.

30. Brasil. Ministério da Saúde. Secretaria de Ciência, Tecnologia e Insumos Estratégicos. Comissão Nacional de Incorporação de Tecnologias no SUS (CONITEC) - Relatório nº 32. Oxigenoterapia domiciliar para o tratamento da Doença Pulmonar Obstrutiva Crônica (DPOC). Brasília (DF): Ministério da Saúde, 2012. Disponível em: <http://conitec.gov.br/images/ Incorporados/Oxigenoterapia-DPOC-final.pdf> Acesso em: 06 abr. 2015.

31. Brasil. Ministério da Saúde. Portaria $n^{\circ}$ 609, de 06 de junho de 2013. Aprova o Protocolo Clínico e Diretrizes Terapêuticas - Doença Pulmonar Obstrutiva Crônica. Diário Oficial da União $2013 ; 07$ jun. 
32. Floriano, L et al. Cuidado realizado pelo cuidador familiar ao idoso dependente, em domicílio, no contexto da estratégia de saúde da família. Texto e Contexto Enfermagem. 2012; 21(3):543-8.

33. Sociedade Brasileira de Pneumologia e Tisiologia: SBPT. II Consenso Brasileiro de Doença Pulmonar Obstrutiva Crônica. Jornal Brasileiro de Pneumologia. 2004; 30(5):1-44.

34. Lynn, I. Oxigenoterapia domiciliar prolongada: uma perspectiva britânica. Jornal de Pediatria. 2011; 87(1):,1-3.

35. Menezes, A et al. The Platino project: methodology of a multicenter prevalence survey of chronic obstructive pulmonary disease in major Latin American cities. BMC MedicalResearchMethodoly. 2004; 4(15):1-7.

36. Global Initiative For Chronic Obstructive Lung Disease: GOLD. Global Strategy for the Diagnosis, Management, and Prevention of COPD - Revised, 2011.Disponível em: $<\mathrm{http}: / / \mathrm{www}$. goldcopd.org/uploads/users/files/GOLD_Report_2011_Feb21.pdf > . Acesso em: 17 jul. 2015.

37. Villas-Bôas, ML; Shimizu, HE; Sanchez, M.N. Clinical and epidemiological profile of patients from the home care program of Federal District Brazil. Journal of Public Health and Epidemiology Review. 2015; 7(6):189-97.

38. Moraes, EN; Moraes, FL; Lima, SP. Características biológicas e psicológicas do envelhecimento. Revista de Medicina de Minas Gerais. 2010; 20(1):67-73.

39. Conselho Municipal De Saúde De Joinville: CMS. Ata da ducentésima décima oitava assembléia geral ordinária do conselho municipal de saúde. Joinville (SC), 2012.

40. Brasil. Lei no 8080, de 19 de setembro de 1990. Dispõe sobre as condições para a promoção, proteção e recuperação da saúde, a organização e o funcionamento dos serviços correspondentes e dá outras providências. Diário Oficial da União 1990; 19 set.

41. Oliveira, L. Responsabilidade municipal pela prestação do serviço de oxigenoterapia domiciliar e seus contornos. Revista de Direito Sanitário. 2009;10(1):39-50.

Artigo apresentado em 02/06/16

Artigo aprovado em 26/09/16

Artigo publicado no sistema em 11/10/16 